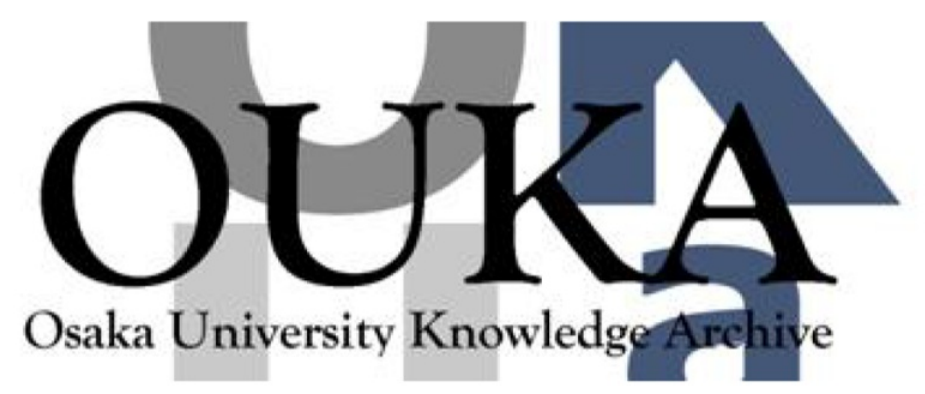

\begin{tabular}{|c|l|}
\hline Title & $\begin{array}{l}\text { Visualization of the moment of mouse sperm-egg } \\
\text { fusion and dynamic localization of IZUM01 }\end{array}$ \\
\hline Author(s) & $\begin{array}{l}\text { Satouh, Yuhkoh; Inoue, Naokazu; Ikawa, Masahito } \\
\text { et al. }\end{array}$ \\
\hline Citation & Journal of Cell Science. 125(21) p. 4985-p. 4990 \\
\hline Issue Date & $2012-11-01$ \\
\hline oaire:version VoR \\
\hline URL & https://hdl. handle. net/11094/78608 \\
\hline rights & $\begin{array}{l}\text { @ 2012. Published by The Company of Biologists } \\
\text { Ltd. }\end{array}$ \\
\hline Note & \\
\hline
\end{tabular}

Osaka University Knowledge Archive : OUKA

https://ir. Library. osaka-u. ac. jp/

Osaka University 


\title{
Visualization of the moment of mouse sperm-egg fusion and dynamic localization of IZUMO1
}

\author{
Yuhkoh Satouh $^{1, *}$, Naokazu Inoue ${ }^{2, \star}$, Masahito Ikawa ${ }^{2}$ and Masaru Okabe $e^{2, \pm}$ \\ ${ }^{1}$ World Premier International Immunology Frontier Research Center, Osaka University, Yamadaoka 3-1, Suita, Osaka 565-0871, Japan \\ ${ }^{2}$ Research Institute for Microbial Diseases, Osaka University, Yamadaoka 3-1, Suita, Osaka 565-0871, Japan \\ *These authors contributed equally to this work \\ ${ }^{\ddagger}$ Author for correspondence (okabe @ biken.osaka-u.ac.jp) \\ Accepted 19 July 2012 \\ Journal of Cell Science 125, 4985-4990 \\ (c) 2012. Published by The Company of Biologists Ltd \\ doi: $10.1242 / j c s .100867$
}

\section{Summary}

Gene disruption experiments have proven that the acrosomal protein IZUMO1 is essential for sperm-egg fusion in the mouse. However, despite its predicted function, it is not expressed on the surface of ejaculated spermatozoa. Here, we report the dynamics of diffusion of IZUMO1 from the acrosomal membrane to the sperm surface at the time of the acrosome reaction, visualized using a fluorescent protein tag. IZUMO1 showed a tendency to localize in the equatorial segment of the sperm surface after the acrosome reaction. This region is considered to initiate fusion with the oolemma. The moment of sperm-egg fusion and the dynamic movements of proteins during fusion were also imaged live. Translocation of IZUMO1 during the fertilization process was clarified, and a fundamental mechanism in mammalian fertilization is postulated.

Key words: Acrosome reaction, Membrane fusion, Protein trafficking, Live-cell imaging, IZUMO1, Sperm-egg fusion

\section{Introduction}

Mammalian fertilization is one form of cell-cell fusion and has been the subject of many studies. Recent experiments using variously prepared unilamellar liposomes have clarified fundamental physicochemical questions in fusion (Brunger et al., 2009; Wessels and Weninger, 2009). Essential factors are also emerging using gene knockout approaches in studies on muscle cell fusion (Abmayr and Pavlath, 2012; Chen, 2011; Powell and Wright, 2011), syncytial formation in the placenta (Dupressoir et al., 2011), fusogens in epithelia and muscle cells in Caenorhabditis elegans (Sapir et al., 2007), and fertilization in nematodes, plants, protists and mammals (Hirai et al., 2008; Ikawa et al., 2010; Nishimura and L'Hernault, 2010), but the mechanisms involved in cell-cell fusion are still not fully explainable on a molecular basis.

The proteins CD9 (Kaji et al., 2000; Le Naour et al., 2000; Miyado et al., 2000) on eggs and IZUMO1 (Inoue et al., 2005) on spermatozoa are the only two factors proven so far to be essential in sperm-egg fusion. IZUMO1 is a protein with a single transmembrane domain and a short cytoplasmic tail. Izumol ${ }^{-/-}$ males are infertile because their spermatozoa are unable to fuse with eggs. However, IZUMO1 is not localized on the plasma membrane of mature spermatozoa (Okabe et al., 1987). Before fusing with eggs, spermatozoa must undergo a physiological change called 'capacitation' and a subsequent morphological change involving restructuring of the sperm membranes and release of the acrosomal contents, called the 'acrosome reaction' (Yanagimachi, 1994). Surprisingly, IZUMO1 was found on the sperm plasma membrane after the acrosome reaction (Inoue et al., 2005). However, how IZUMO1 moves to the plasma membrane and how it behaves during sperm-egg fusion are questions that remain to be elucidated.

We produced a transgenic mouse line that expressed the IZUMO1-mCherry fusion protein (Red-IZUMO1). This transgenic mouse line was crossed with another transgenic mouse line that expresses green fluorescent protein (GFP) in the sperm acrosome (Nakanishi et al., 1999), so that the acrosomal status could be monitored in living spermatozoa using fluorescence microscopy. Using this double transgenic mouse line, changes in the location of IZUMO1 during the acrosome reaction and the behavior of IZUMO1 at the moment of sperm-egg fusion were monitored

\section{Results and Discussion}

Visualization of IZUMO1 by tagging with mCherry

To visualize the translocation of IZUMO1 during fertilization in live spermatozoa, we generated a transgenic mouse line expressing mCherry-tagged IZUMO1 (Red-IZUMO1) using a calmegin promoter (Watanabe et al., 1995). The expression of RedIZUMO1 on sperm was confirmed by western blotting using anti-IZUMO1 and anti-mCherry antibodies (Fig. 1A). The resulting Red-IZUMO1 was proven to be biologically functional by gene rescue experiments. When Red-IZUMO1 was introduced into an Izumol $^{-1-}$ background, the infertile phenotype was rescued to the wild-type level. The average litter size obtained was $10.0 \pm 1.8$ pups (mean \pm s.d., $n=10$ ), while wild-type males with a similar genetic background produced $8.7 \pm 2.5$ pups $(n=13)$. The present experiments were carried out using Red-IZUMOI mice with an Izumol $^{-1-}$ genetic background unless otherwise stated.

The localization of IZUMO1 in live spermatozoa before the acrosome reaction was investigated using the Red-IZUMOI transgenic mice, crossed with another transgenic mouse line that expresses GFP in the acrosome (Green-Ac) (Nakanishi et al., 1999) (Fig. 1B).

IZUMO1 is not detectable by antibodies in living spermatozoa before the acrosome reaction (Okabe et al., 1987; Inoue et al., 
A
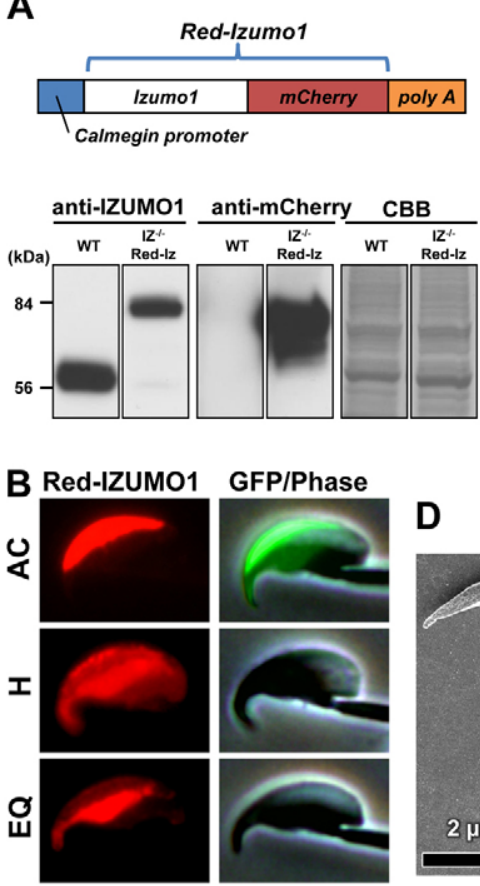

D
C

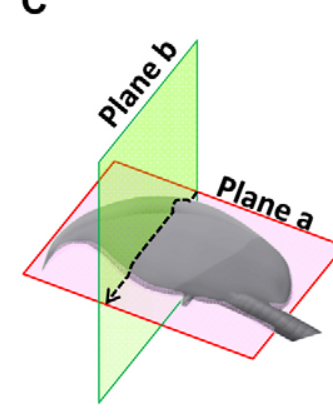

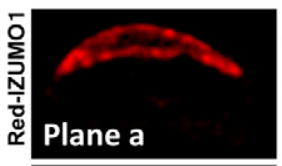

\section{Plane b}

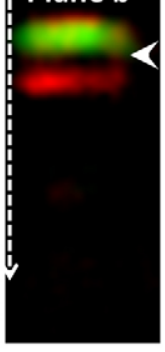

Red-IZUMO1:Green-Ac sperm
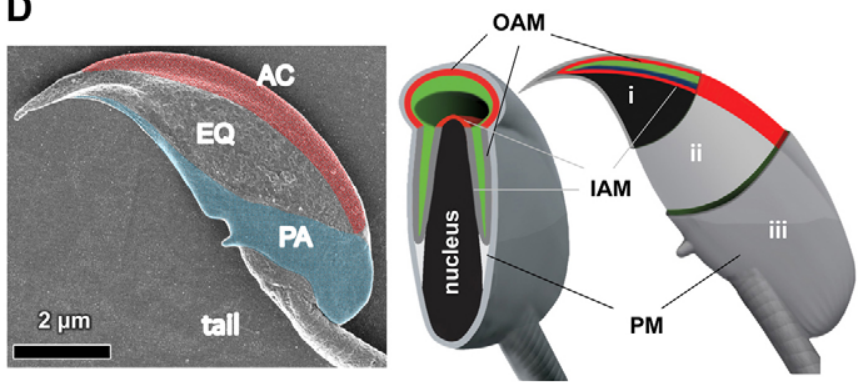

Fig. 1. Tagging of IZUMO1 with mCherry and acrosomal status-dependent distribution. (A) A calmegin-promoter-driven Izumol cDNA, tagged by mCherry, was constructed (Red-IZUMOI) and transgenic mice were produced. Red-IZUMO1 in spermatozoa was detected as an 84-kDa band by mAb No. 125 anti-IZUMO1 and No. 632397 anti-mCherry antibodies. CBB, Coomassie brilliant blue staining. (B) Fluorescent images of spermatozoa from a 'RedIZUMO1:Green-Ac' double transgenic mouse. GFP-positive, acrosome-intact spermatozoa had Red-IZUMO1 in the acrosomal cap (AC) area, whereas RedIZUMO1 spread to the entire head $(\mathrm{H})$ or to the equatorial segment (EQ) in GFP-negative, acrosome-reacted spermatozoa. (C) Three-dimensional confocal images of Red-IZUMO1 (red) and GFP (green) in an acrosome-intact spermatozoon. A GFP-depleted area (arrowhead) was found between the inner acrosomal membrane and a GFP-abundant area in the acrosomal matrix (see also supplementary material Movies 1 and 2). (D) Schematic diagram showing the three distinctively different areas overlapping with a scanning electron micrographic view of the sperm head (left). Note that Red-IZUMO1 was localized in both the outer and inner acrosomal membrane in the AC but not in the equatorial segment. Areas (i), (ii) and (iii) indicate a transverse section, an outer acrosomal-membrane-level view and plasma-membrane-level view of a spermatozoon, respectively. AC, EQ and PA indicate the acrosomal cap, equatorial segment and postacrosomal region, respectively. IAM, OAM and PM indicate the inner acrosomal, outer acrosomal and plasma membranes, respectively.

2005). Therefore, we first estimated the localization of RedIZUMO1 using a laser confocal microscope with a high magnification $(\times 150)$ objective lens. As shown in threedimensional images, Red-IZUMO1 was localized exclusively in acrosomal cap region of both the inner and outer acrosomal membranes (Fig. 1C). Supplementary material Movies 1 and 2 show the distributions of Red-IZUMO1 and GFP, in sections through orthogonal planes ' $a$ ' and ' $b$ ', respectively.

Despite its very soluble nature, the transgenically expressed GFP was observed exclusively in the outer region of the acrosome (Fig. 1C,D). This might have been associated with the differential release of soluble and matrix components that occurs during the acrosome reaction (Harper et al., 2008; Kim and Gerton, 2003) and to the layered acrosomal structure shown by electron microscopy (Foster et al., 1997).

\section{Translocation of IZUMO1 during the acrosome reaction}

In the next experiment, we aimed to track the movement of IZUMO1 during the acrosome reaction. Because mouse spermatozoa tend to die during observation using a normal confocal fluorescence microscope, we used a low-invasive Nipkow-disk confocal microscope live imaging system equipped with an electron multiplying charge-coupled device (EM-CCD) camera (Yamagata et al., 2009) (Fig. 2A).
To observe the acrosome reaction in live spermatozoa, we used an isolated mouse zona pellucida to trap them. Zona-bound spermatozoa were incubated in a chamber situated on the stage of a confocal microscope at $37^{\circ} \mathrm{C}$ under $5 \% \mathrm{CO}_{2}$ in air and were photographed periodically. A representative time-lapse observation of the acrosome reaction is shown in Fig. $2 \mathrm{~B}$ and in supplementary material Movie 3, which depicts the disappearance of GFP between frames 4:43 (4 min and $43 \mathrm{~s}$ ) and 5:00. Most Red-IZUMO1 apparently remained in the acrosomal cap region, with part seemingly diffused over the entire head at frame 5:00. This translocation subsided by frame 5:33. At the time of the acrosome reaction, the outer acrosomal and plasma membranes fuse to form pores and allow exocytosis of the acrosomal contents. This was when IZUMO1 translocate from the acrosomal membrane to the plasma membrane (Fig. 2B,C), coincidentally with the disappearance of GFP from the acrosome. The total amount of Red-IZUMO1 fluorescence did not alter significantly following translocation to the plasma membrane (Fig. 2D).

One of the characteristics of Red-IZUMO1 is its affinity for the equatorial segment (Fig. 2B). Red-IZUMO1; after it had spread out to the entire head (' $\mathrm{H}$ '-type distribution), it showed a tendency to gather in the equatorial segment ('EQ' type). Another sperm protein, ADAM1B, is contained in the postacrosomal area and is not able to diffuse over the border to the equatorial 
A

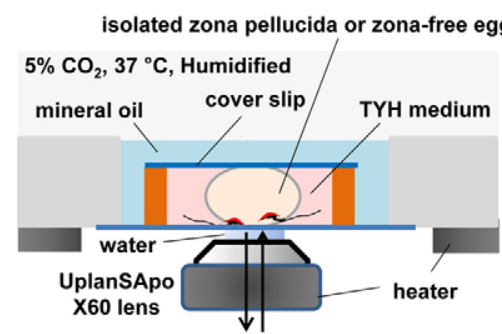

spinning-disk confocal laser scanning system with a high sensitivity EM-CCD camera

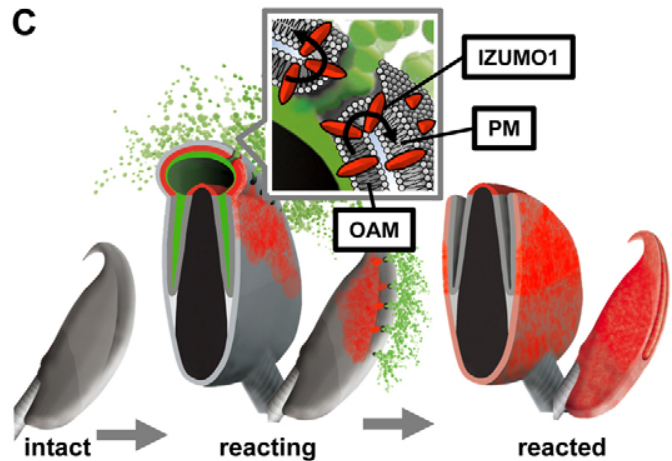

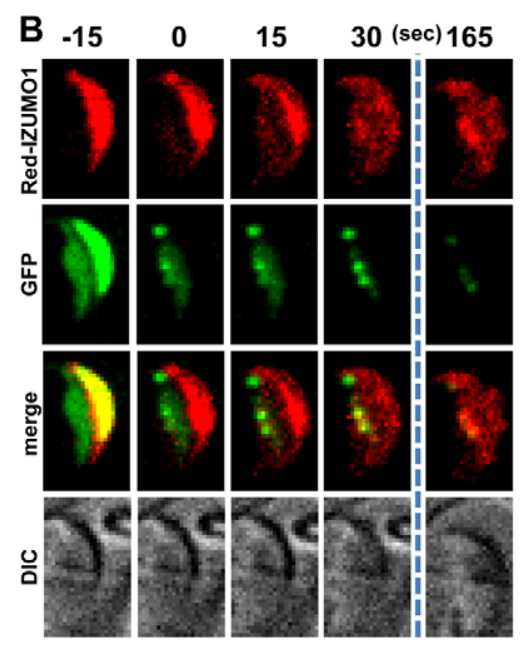

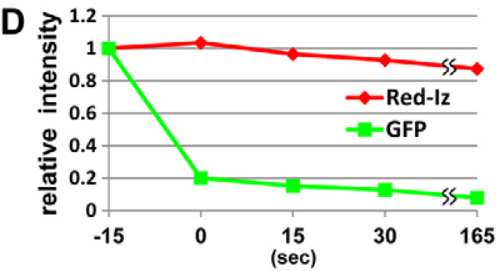

Fig. 2. Translocation of Red-IZUMO1 from the outer acrosomal membrane to the sperm plasma membrane. (A) Acrosome reactions were observed using a warmed chamber (Olympus, MI-IBC) with a heater-equipped water immersion lens (Olympus, UPLSAPO 60XW). Spermatozoa were trapped on an isolated zona pellucida (see Materials and Methods). The fluorescent image was taken using a spinning-disk confocal laser scanning system (Yokogawa, CSU-X1) with a highly sensitive EMCCD camera (Andor, iXon plus; see Materials and Methods). (B) A representative time-lapse image of Red-IZUMO1 translocation at the time of acrosomal exocytosis detected by GFP fluorescence. From the time of GFP dispersal, it took less than $30 \mathrm{~s}$ for Red-IZUMO1 to spread across the entire head (see also supplementary material Movie 3). (C) Schematic diagram showing the estimated translocation pathway of RedIZUMO1 from the acrosomal membrane to the sperm plasma membrane. The inset shows the hypothetical movement of IZUMO1 in this event. (D) The fluorescence intensities from mCherry (Red-IZUMO1) and GFP (acrosomal contents) during the acrosome reaction. The total amount of Red-IZUMO1 was almost the same even after the acrosome reaction.

A

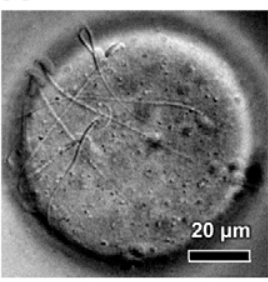

B

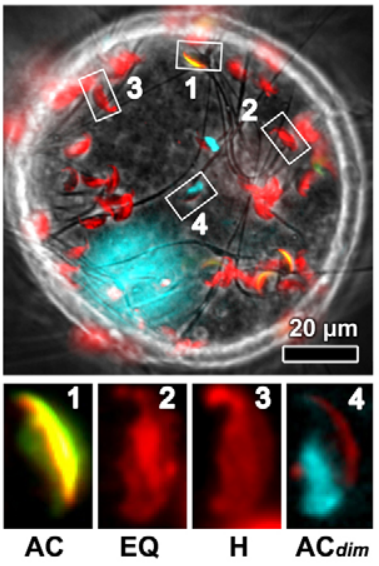

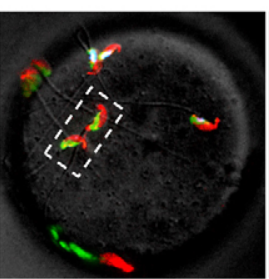

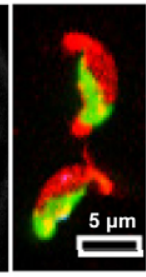

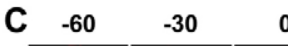
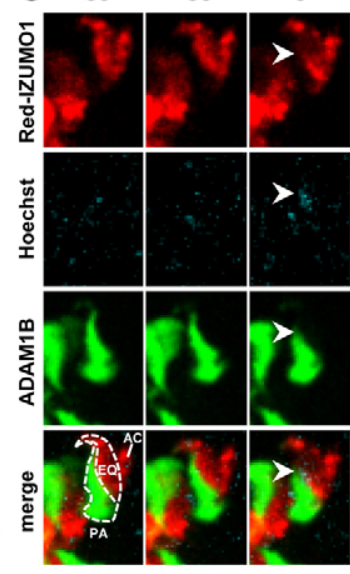

.

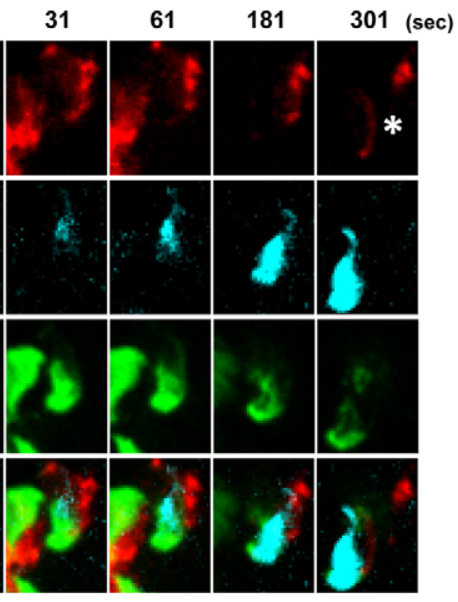

Fig. 3. Diffusion of Red-IZUMO1 at the time of sperm-egg fusion. (A) Most spermatozoa collected from the perivitelline space $8 \mathrm{~h}$ after copulation from $C d 9^{-/-}$females mated with Red-IZUMOI males were resistant to staining with Hoechst 33258 (cyan), indicating that they were still alive after penetrating the zona pellucida. Staining with the Alexa-488-conjugated mAb No. 107.57 antibody showed that ADAM1B was exclusively localized in the postequatorial segment of the spermatozoa (green). A magnified image of EQ-type sperm from the boxed area is shown on the right. Because sperm-egg fusion was blocked by using $C d 9^{-/-}$eggs, no spermatozoa showed the $\mathrm{AC}_{d i m}$ pattern. (B) Acrosome-intact (GFP-positive) spermatozoa on the egg plasma membrane always showed an acrosomal cap (AC) pattern, whereas the acrosome-reacted spermatozoa and spermatozoa imaged before fusion showed Red-IZUMO1 spread out to the entire head ('H'-type distribution), or with a tendency to gather in the equatorial segment ('EQ' type). Fused spermatozoa (Hoechst 33342-positive uptake from the preloaded oolemma) showed the dim acrosomal cap pattern ( $\mathrm{AC}_{\text {dim }}$ ) without exception. $(\mathrm{C}) \mathrm{A}$ representative time-lapse view of sperm-egg fusion. The initiation of fusion (time 0) was detected by diffusion of Red-IZUMO1 from the equatorial segment and the concomitant transfer of Hoechst 33342 dye to sperm in the same area (indicated by arrowheads). The diffusion of membrane in the postacrosomal area identified by ADAM1B (green) started about $60 \mathrm{~s}$ after the initiation of sperm-egg fusion and was accompanied by expansion of the Hoechst 33342 staining area toward the posterior head. The asterisk indicates the emergence of the $\mathrm{AC}_{\text {dim }}$ pattern. The sperm head localization drifted during the fusion process and an analysis is shown in supplementary material Fig. S1. 

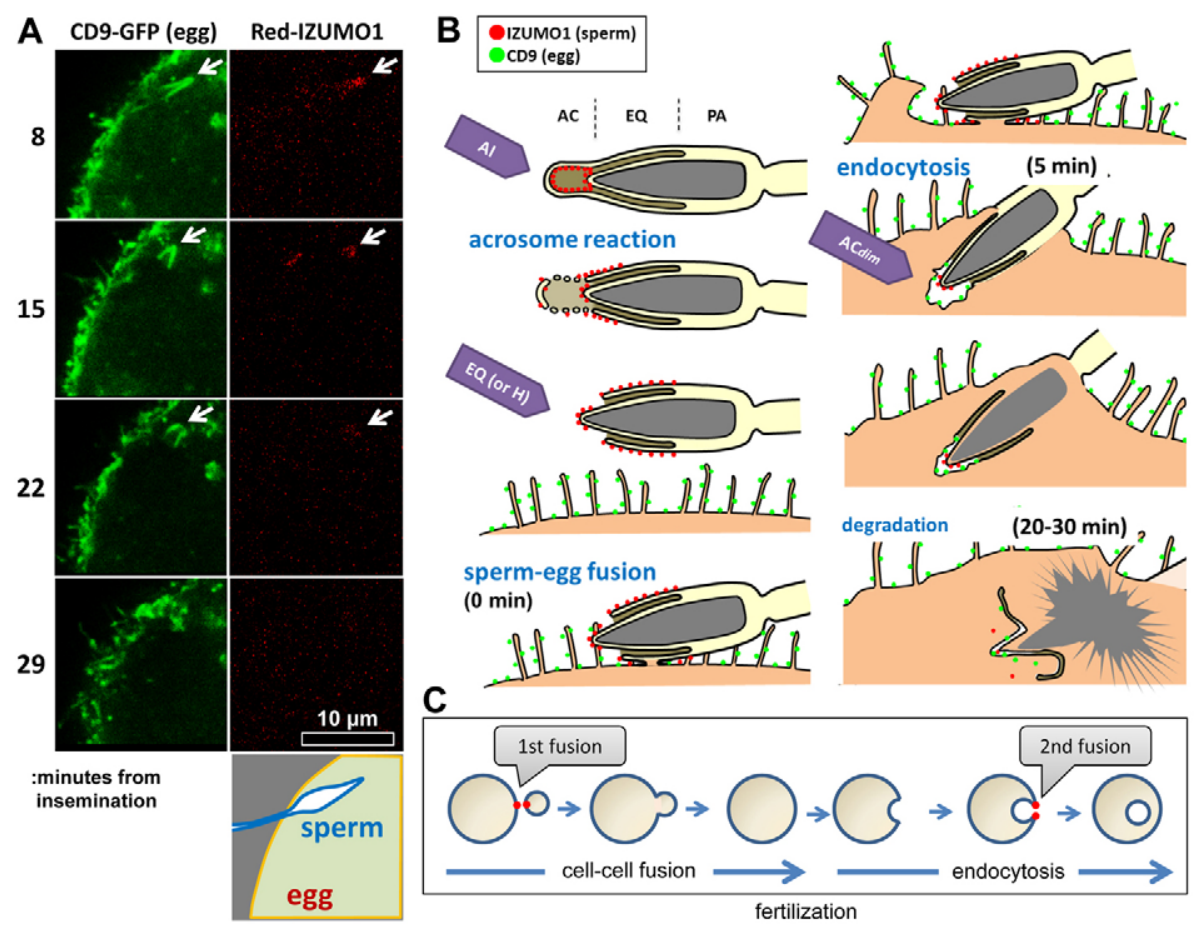

Fig. 4. The fate of IZUMO1 during the acrosome reaction and following sperm-egg fusion.

(A) Time-lapse confocal images of a fertilized egg expressing CD9-GFP. Eggs were inseminated and mounted on the observation chamber. It took $8 \mathrm{~min}$ to start the imaging after the insemination. Internalization of the membrane had already started by this time. The internalized wedge-shaped vesicle was deformed and Red-IZUMO1 staining disappeared within $30 \mathrm{~min}$ of insemination. (B) IZUMO1 was localized initially on the inner and outer acrosomal membrane and translocated to the plasma membrane at the time of the acrosome reaction. Acrosome-reacted spermatozoa have a complicated invaginated membrane structure. Fusion started at the equatorial segment and IZUMO1 dispersed onto the egg plasma membrane. A part of the sperm membrane including the inner acrosomal membrane is routinely endocytosed by the egg and the $\mathrm{AC}_{\text {dim }}$ pattern of IZUMO1 formed at this stage. (C) Fertilization consists of two distinct fusion processes. First, cell-cell fusion combines two independent membranes (sperm and egg) into one. Second, endocytosis divides this single membrane into two parts: one of them invaginates and the other remains on the egg surface. Red dots indicate where the fusion occurs.

segment (Kim et al., 2003). However, this border did not seem to block the translocation of Red-IZUMO1. A testis-specific serine kinase (Tssk6) is reported to be involved in the translocation of IZUMO1 (Sosnik et al., 2009), but the precise driving force and mechanism involved have yet to be clarified.

\section{Diffusion of IZUM01 to the egg plasma membrane}

$C d 9^{-/-}$eggs are known to have a defect in their ability to fuse with spermatozoa (Kaji et al., 2000; Le Naour et al., 2000; Miyado et al., 2000). When mixed with wild-type spermatozoa, many fusioncompetent spermatozoa were observed in the perivitelline space of $C d 9^{-/-}$eggs (Inoue et al., 2011). We observed the localization of IZUMO1 in more than 200 of these spermatozoa and all of them showed EQ- or H-type fluorescence patterns (Fig. 3A). This suggests that such patterns are typical for spermatozoa before fusion.

In the next experiment, we preincubated wild-type eggs with cell-permeable Hoechst 33342 and allowed the egg cytoplasm to accumulate this dye. Spermatozoa were then added to the eggs. In this condition, only egg-fused sperm nuclei become stained with Hoechst 33342 (Inoue et al., 2005). These fused spermatozoa showed IZUMO1 localization in their acrosomal cap area with a dim fluorescence $\left(\mathrm{AC}_{\text {dim }}\right.$ pattern) without exception (Fig. 3B; supplementary material Table S1).

By live imaging using transgenic mouse spermatozoa, it was indicated that fusion started from the equatorial segment and proceeded to the posterior part of the sperm head, as the dispersal of ADAM1B to this area (Hunnicutt et al., 2008) did not take place until all the Red-IZUMO1 in the equatorial segment had diffused away (Fig. 3C). AC dim pattern appeared after RedIZUMO1 from the equatorial segment diffused to the egg plasma membrane (Fig. 3C). In combination with the observation shown in Fig. $4 \mathrm{~A}, \mathrm{AC}_{\text {dim }}$ pattern was indicated to derive from RedIZUMO1 initially resided in the acrosomal cap area of inner acrosomal membrane. The diffusion of Hoechst 33342 to the sperm nucleus started in the equatorial segment, where the disappearance of Red-IZUMO1 was first observed, progressing then to the posterior direction and finally to the anterior part. It took about $5 \mathrm{~min}$ for spermatozoa to proceed from the initiation of fusion to the latter stage (Fig. 3C; supplementary material Movie 4). Spermatozoa that did not take up the Hoechst dye failed to show diffusion of either Red-IZUMO1 or ADAM1B.

\section{Internalization of inner acrosomal membrane after fusion}

After fusion with an egg in the equatorial segment, the sperm membrane comprises a continuous single membrane plane with a complicated invaginated structure. However, sperm-egg fusion is not fully accomplished at this point, as electron microscopic observation has indicated the "internalization" of the invaginated inner acrosomal membrane occurs later in the fertilization process (Bedford et al., 1979; Huang and Yanagimachi, 1985).

When CD9-GFP eggs (Miyado et al., 2008) were fertilized by Red-IZUMO1 spermatozoa, a wedge-shaped vesicle that included both sperm and egg membranes was found to form the $\mathrm{AC}_{\text {dim }}$ pattern (Fig. 4A). Because the $\mathrm{AC}_{\text {dim }}$ pattern was observed in all fusing spermatozoa, this internalization, which was shown to take about 5 min, was an essential process for fertilization (Fig. 4B).

It should be noted that the "internalization" process also requires membrane fusion. The principle of this fusion is to divide a single membrane into two separate membranes in contrast to the initial sperm-egg fusion, which joins two membranes into one (Fig. 4C; supplementary material Figs S2, S3).

These dynamic movements of the sperm and egg membranes during fertilization, which were demonstrated by gene-manipulated animals, will help elucidate the mechanisms of mammalian fertilization.

\section{Materials and Methods}

Animals and antibodies

All of the animal experiments were performed with the approval of the Animal Care and Use Committee of Osaka University. Izumol ${ }^{-1-}$ and B6;C3 Tg(acro3-EGFP)01Osb 
transgenic mouse lines were generated as described (Inoue et al., 2005; Nakanishi et al., 1999). The $C d 9^{-/-}$mouse line (Miyado et al., 2000) was a gift from E. Mekada of Biken. Anti-ADAM1B monoclonal antibody (mAb No. 107.57), generated as described (Ikawa et al., 2011), was conjugated with Alexa Fluor 488 using an antibody labeling kit (Life Technologies, Carlsbad, CA, USA).

\section{Generation of Red-IZUMO1 transgenic mice}

A construct was prepared in the pBluescript SK II+ plasmid. We designed a testisspecific expression construct inserting Izumol cDNA conjugated with mCherry (derived from the pmCherry-N1 vector; Takara Bio Inc., Shiga, Japan) at the Cterminal between the calmegin promoter and a rabbit beta-globin polyadenylation signal (Red-IZUMO1: Fig. 1A). Transgenic mouse lines were produced by injecting $3.0 \mathrm{~kb}$ AseI-SalI DNA fragments into the pronuclei of Izumol ${ }^{+/-}$male $\times$Izumol $^{-1}$ female fertilized eggs. After transfer of the embryos to pseudopregnant foster mothers, we obtained nine pups that were demonstrated to have the transgene by polymerase chain reaction (PCR) amplification. Offspring carrying the transgene were identified by PCR using primers A (5'-CCTTCCTGCGGCTTGTTCTCT-3') and $\mathrm{B}\left(5^{\prime}\right.$-GGTCTCAGAACTTTGCTCCCAAACCCTGTA- $\left.3^{\prime}\right)$ for the transgene of Izumo 1-mCherry. The endogenous Izumol and their mutated alleles were detected by PCR using primers C (5'-GGGTTCACTCTCCAGCTACCCCAAACTCAC-3') and D (5'-CAGAACCCCGAACCCAGCCTATGCC- $\left.3^{\prime}\right)$ and primers E (5'GCTTGCCGAATATCATGGTGGAAAATGGCC-3') and D, respectively. Among these lines, one transgenic line retained the brightest red fluorescence derived from mCherry on the sperm head and was used in all analyses.

\section{Immunoblot analysis}

Immunoblot analysis was performed as described previously (Inoue et al., 2008) Samples were subjected to sodium dodecyl sulfate PAGE followed by western blotting under reduced conditions. For IZUMO1, antibody mAb No. 125 was used For mCherry, a cross-reacting antibody against red fluorescent protein No. 632397 (Becton Dickinson and Co., Franklin Lakes, NJ) was used.

\section{Three-dimensional image reconstruction of Red-IZUMO1:Green-AC} spermatozoa

Deconvolution analysis was performed on confocal Z-stacks $(0.1 \mu \mathrm{m}$ optical thickness) by using the Iterative Deconvolve 3D plugin of ImageJ software (http://rsbweb.nih.gov/ ij/). An oil-immersion, high-magnification objective lens (UAPON 150XOTIRF, Olympus, Tokyo, Japan) was used for Nipkow-disk confocal microscopy.

\section{Time-lapse imaging of acrosome reactions on the zona pellucida}

The ooplasm was flushed out from the zona pellucida using a piezo manipulator (Yamagata et al., 2002) and the empty zonae were collected. Spermatozoa from the Red-IZUMO1:Green-Ac double transgenic mouse line were preincubated for $90 \mathrm{~min}$ in TYH medium (Toyoda et al., 1971) and were added to the empty zonae at a final concentration of $2 \times 10^{5}$ cell $/ \mathrm{ml}$. After $3 \mathrm{~min}$, the spermatozoa on the zonae were transferred to an observation chamber with a glass-bottomed dish and were gently pressed down with a coverslip. The chamber was set on an inverted microscope equipped with a noninvasive Nipkow-disk confocal system (Yamagata et al., 2009) and confocal images were taken every $15 \mathrm{~s}$ for $50 \mathrm{~min}$ under three different laser excitations $(405,488$ and $561 \mathrm{~nm})$. At each time point, five images with a different $z$-axis plane $(1 \mu \mathrm{m}$ increments $)$ from the bottom were photographed. A water-immersion objective lens (UPLSAPO 60XW, Olympus, Tokyo, Japan) was used. Dead spermatozoa were identified and excluded from the analysis by DNA staining with the cell-impermeable Hoechst 33258 (blue) dye.

Observation of sperm-egg fusion by dye transfer

Hoechst dye transfer from B6D2F1 eggs to Red-IZUMO1:Green-Ac spermatozoa was observed as described (Inoue et al., 2005) without using any fixative.

\section{Observation of Red-IZUMO1 spermatozoa in the perivitelline space of} $\mathrm{CdO}^{-1-}$ eggs

$C d 9^{-/}$females were mated with Red-IZUMO1 males $12 \mathrm{~h}$ after an hCG injection for ovulation induction. Vaginal plug formation was examined every $15 \mathrm{~min}$ and eggs were recovered from the $C d 9^{---}$females $8 \mathrm{~h}$ after coitus. Cumulus cells were removed by hyaluronidase treatment and dead cells were identified by incubation with cell-impermeable Hoechst 33528 dye for 15 min. Spermatozoa in the perivitelline space were stained (or not stained) with an Alexa-488-labeled antiADAM1B antibody ( $\mathrm{mAb}$ No. 107.57) for $15 \mathrm{~min}$ and the eggs were observed using confocal microscopy after being pressed gently between the observation dish and a coverslip (Fig. 2A)

\section{Time-lapse imaging of sperm-egg fusion}

Zona-free and Hoechst 33342-loaded B6D2F1 eggs were prepared as described above. Epididymal spermatozoa were collected from males and preincubated in TYH medium with the anti-ADAM1B antibody for $90 \mathrm{~min}$ and were then added to the zona-free eggs at a final concentration of $2 \times 10^{5}$ spermatozoa/ml. After incubation for $2 \mathrm{~min}$, the eggs were transferred to $3 \mu \mathrm{l}$ TYH drops in an observation chamber as described above, except that four blobs of Vaseline grease were placed around the eggs to prevent them from being squashed. Confocal microscopy images were taken every $30 \mathrm{~s}$ for $50 \mathrm{~min}$ under three different laser wavelength excitations (405, 488 and $561 \mathrm{~nm}$ ). At each time point, 11 images with different $z$-axis planes (in $0.5 \mu \mathrm{m}$ increments) were photographed.

\section{Colocalization of CD9 with internalizing IZUMO1 signals on the inner} acrosomal membrane

Fertilization between Hoechst 33342-loaded zona-free $C d 9^{-/} ; C D 9-G F P$ eggs and Red-IZUMO1 spermatozoa was observed. After incubation with spermatozoa for $5 \mathrm{~min}$, the eggs were washed briefly and observed. Confocal images of whole eggs with different $z$-axis planes (in $1 \mu \mathrm{m}$ increments) were taken every $7.5 \mathrm{~min}$ for $45 \mathrm{~min}$

\section{Acknowledgements}

We are grateful to Professor Ryuzo Yanagimachi for his invaluable advice during the preparation of this manuscript. We thank Kazuo Yamagata of RIKEN, CDB, for technical advice concerning use of a Nipkow-disk confocal microscope, Yoko Esaki and Yumiko Koreeda for technical assistance with producing transgenic mouse lines and Jooeun Lee for drawing the illustrations.

\section{Funding}

This work was supported by grants from the Ministry of Education, Culture, Sports, Science and Technology of Japan.

Supplementary material available online at http://jcs.biologists.org/lookup/suppl/doi:10.1242/jcs.100867/-/DC1

\section{References}

Abmayr, S. M. and Pavlath, G. K. (2012). Myoblast fusion: lessons from flies and mice. Development 139, 641-656.

Bedford, J. M., Moore, H. D. and Franklin, L. E. (1979). Significance of the equatorial segment of the acrosome of the spermatozoon in eutherian mammals. Exp. Cell Res. 119, 119-126.

Brunger, A. T., Weninger, K., Bowen, M. and Chu, S. (2009). Single-molecule studies of the neuronal SNARE fusion machinery. Annu. Rev. Biochem. 78, 903-928.

Chen, E. H. (2011). Invasive podosomes and myoblast fusion. Curr. Top. Membr. 68, 235-258.

Dupressoir, A., Vernochet, C., Harper, F., Guégan, J., Dessen, P., Pierron, G. and Heidmann, T. (2011). A pair of co-opted retroviral envelope syncytin genes is required for formation of the two-layered murine placental syncytiotrophoblast. Proc Natl. Acad. Sci. USA 108, E1164-E1173.

Foster, J. A., Friday, B. B., Maulit, M. T., Blobel, C., Winfrey, V. P., Olson, G. E. Kim, K. S. and Gerton, G. L. (1997). AM67, a secretory component of the guinea pig sperm acrosomal matrix, is related to mouse sperm protein sp56 and the complement component 4-binding proteins. J. Biol. Chem. 272, 12714-12722.

Harper, C. V., Cummerson, J. A., White, M. R., Publicover, S. J. and Johnson, P. M. (2008). Dynamic resolution of acrosomal exocytosis in human sperm. J. Cell Sci. 121, 2130-2135.

Hirai, M., Arai, M., Mori, T., Miyagishima, S. Y., Kawai, S., Kita, K., Kuroiwa, T., Terenius, O. and Matsuoka, H. (2008). Male fertility of malaria parasites is determined by GCS1, a plant-type reproduction factor. Curr. Biol. 18, 607613.

Huang, T. T., Jr and Yanagimachi, R. (1985). Inner acrosomal membrane of mammalian spermatozoa: its properties and possible functions in fertilization. Am. J. Anat. 174, 249-268.

Hunnicutt, G. R., Koppel, D. E., Kwitny, S. and Cowan, A. E. (2008). Cyclic 3',5'AMP causes ADAM1/ADAM2 to rapidly diffuse within the plasma membrane of guinea pig sperm. Biol. Reprod. 79, 999-1007.

Ikawa, M., Inoue, N., Benham, A. M. and Okabe, M. (2010). Fertilization: a sperm's journey to and interaction with the oocyte. J. Clin. Invest. 120, 984-994.

Ikawa, M., Tokuhiro, K., Yamaguchi, R., Benham, A. M., Tamura, T., Wada, I., Satouh, Y., Inoue, N. and Okabe, M. (2011). Calsperin is a testis-specific chaperone required for sperm fertility. J. Biol. Chem. 286, 5639-5646.

Inoue, N., Ikawa, M., Isotani, A. and Okabe, M. (2005). The immunoglobulin superfamily protein Izumo is required for sperm to fuse with eggs. Nature 434, 234 238.

Inoue, N., Ikawa, M. and Okabe, M. (2008). Putative sperm fusion protein IZUMO and the role of $N$-glycosylation. Biochem. Biophys. Res. Commun. 377, 910-914.

Inoue, N., Satouh, Y., Ikawa, M., Okabe, M. and Yanagimachi, R. (2011). Acrosomereacted mouse spermatozoa recovered from the perivitelline space can fertilize other eggs. Proc. Natl. Acad. Sci. USA. 108, 20008-20011.

Kaji, K., Oda, S., Shikano, T., Ohnuki, T., Uematsu, Y., Sakagami, J., Tada, N., Miyazaki, S. and Kudo, A. (2000). The gamete fusion process is defective in eggs of Cd9-deficient mice. Nat. Genet. 24, 279-282. 
Kim, K. S. and Gerton, G. L. (2003). Differential release of soluble and matrix components: evidence for intermediate states of secretion during spontaneous acrosomal exocytosis in mouse sperm. Dev. Biol. 264, 141-152.

Le Naour, F., Rubinstein, E., Jasmin, C., Prenant, M. and Boucheix, C. (2000). Severely reduced female fertility in CD9-deficient mice. Science 287, 319-321.

Miyado, K., Yamada, G., Yamada, S., Hasuwa, H., Nakamura, Y., Ryu, F., Suzuki, K., Kosai, K., Inoue, K., Ogura, A. et al. (2000). Requirement of CD9 on the egg plasma membrane for fertilization. Science 287, 321-324.

Miyado, K., Yoshida, K., Yamagata, K., Sakakibara, K., Okabe, M., Wang, X., Miyamoto, K., Akutsu, H., Kondo, T., Takahashi, Y. et al. (2008). The fusing ability of sperm is bestowed by CD9-containing vesicles released from eggs in mice. Proc. Natl. Acad. Sci. USA 105, 12921-12926.

Nakanishi, T., Ikawa, M., Yamada, S., Parvinen, M., Baba, T., Nishimune, Y. and Okabe, M. (1999). Real-time observation of acrosomal dispersal from mouse sperm using GFP as a marker protein. FEBS Lett. 449, 277-283.

Nishimura, H. and L'Hernault, S. W. (2010). Spermatogenesis-defective (spe) mutants of the nematode Caenorhabditis elegans provide clues to solve the puzzle of male germline functions during reproduction. Dev. Dyn. 239, 1502-1514.

Okabe, M., Adachi, T., Takada, K., Oda, H., Yagasaki, M., Kohama, Y. and Mimura, T. (1987). Capacitation-related changes in antigen distribution on mouse sperm heads and its relation to fertilization rate in vitro. J. Reprod. Immunol. 11, 91100 .
Powell, G. T. and Wright, G. J. (2011). Jamb and jamc are essential for vertebrate myocyte fusion. PLoS Biol. 9, e1001216.

Sapir, A., Choi, J., Leikina, E., Avinoam, O., Valansi, C., Chernomordik, L. V., Newman, A. P. and Podbilewicz, B. (2007). AFF-1, a FOS-1-regulated fusogen, mediates fusion of the anchor cell in C. elegans. Dev. Cell 12, 683-698.

Sosnik, J., Miranda, P. V., Spiridonov, N. A., Yoon, S. Y., Fissore, R. A., Johnson, G. R. and Visconti, P. E. (2009). Tssk6 is required for Izumo relocalization and gamete fusion in the mouse. J. Cell Sci. 122, 2741-2749.

Toyoda, Y., Yokoyama, M. and Hoshi, T. (1971). Studies on the fertilization of mouse eggs in vitro. Jpn. J. Anim. Reprod. 16, 152-157.

Watanabe, D., Okabe, M., Hamajima, N., Morita, T., Nishina, Y. and Nishimune, Y. (1995). Characterization of the testis-specific gene 'calmegin' promoter sequence and its activity defined by transgenic mouse experiments. FEBS Lett. 368, 509-512.

Wessels, L. and Weninger, K. (2009). Physical aspects of viral membrane fusion. ScientificWorldJournal 9, 764-780.

Yamagata, K., Nakanishi, T., Ikawa, M., Yamaguchi, R., Moss, S. B. and Okabe, M. (2002). Sperm from the calmegin-deficient mouse have normal abilities for binding and fusion to the egg plasma membrane. Dev. Biol. 250, 348-357.

Yamagata, K., Suetsugu, R. and Wakayama, T. (2009). Long-term, six-dimensional live-cell imaging for the mouse preimplantation embryo that does not affect full-term development. J. Reprod. Dev. 55, 343-350.

Yanagimachi, R. (1994). Mammalian fertilization. In: The Physiology of Reproduction, 2nd edition (ed. E. Knobil and J. D. Neil), pp. 189-317. New York, NY: Raven Press. 

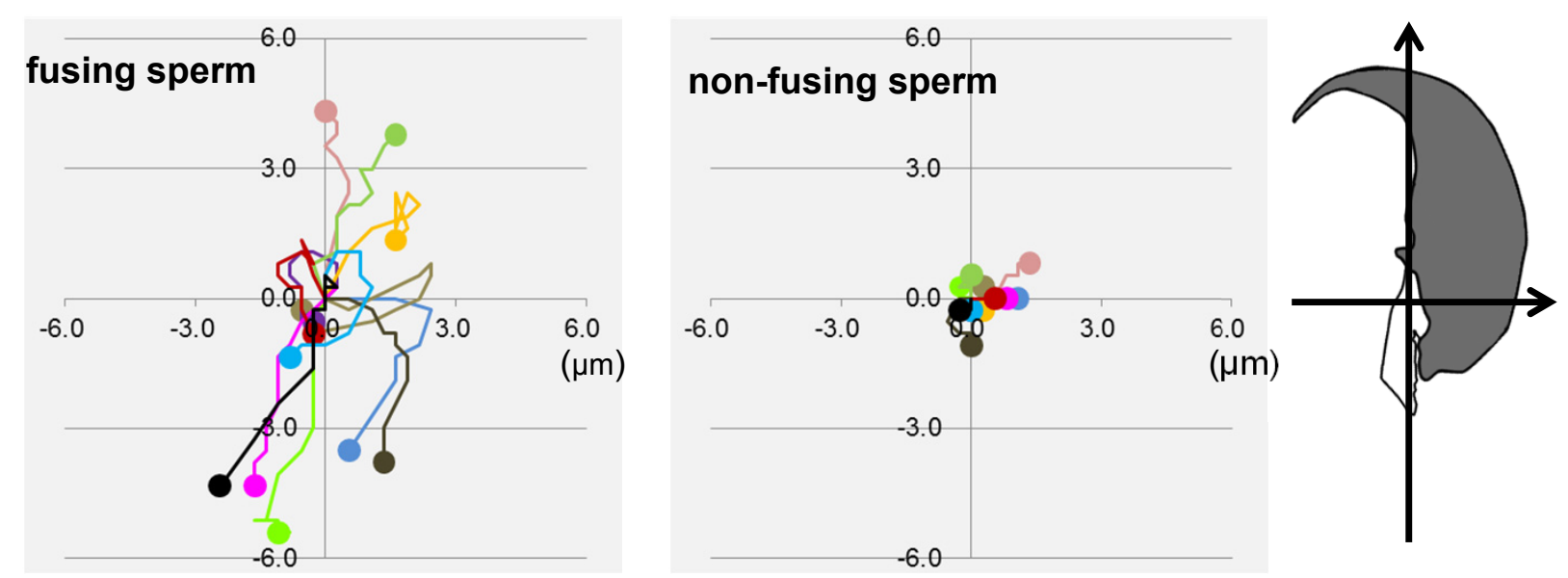

B $(\mu \mathrm{m})$

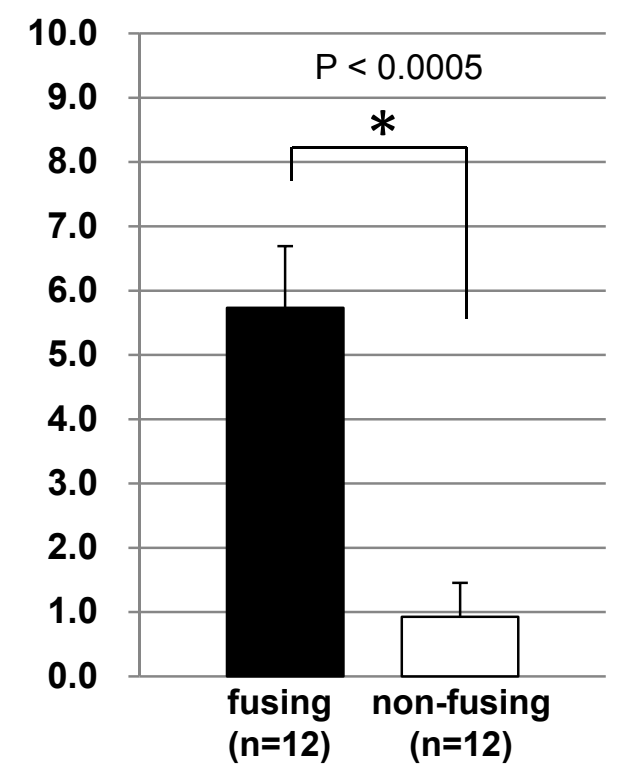

Figure S1. The fusing point drifts during the sperm-egg fusion process.

(A) The neck point of sperm head was plotted in the center with the sperm head to the 12 o'clock direction. Then the same neck point of each fusing sperm (left figure) or non-fusing sperm (right figure) was plotted every 30 seconds for a further 5 minutes. The direction of the drift showed the tendency towards the antero-posterior axes rather than the left-right axes. (B) The total distance which sperm drifted. The fusing sperm drifted a significantly greater distance $(5.73 \mu \mathrm{m} \pm 0.96$; mean $\pm \mathrm{SD})$ than the sperm that bound on the egg surface $(0.93 \mu \mathrm{m} \pm 0.53)$. 

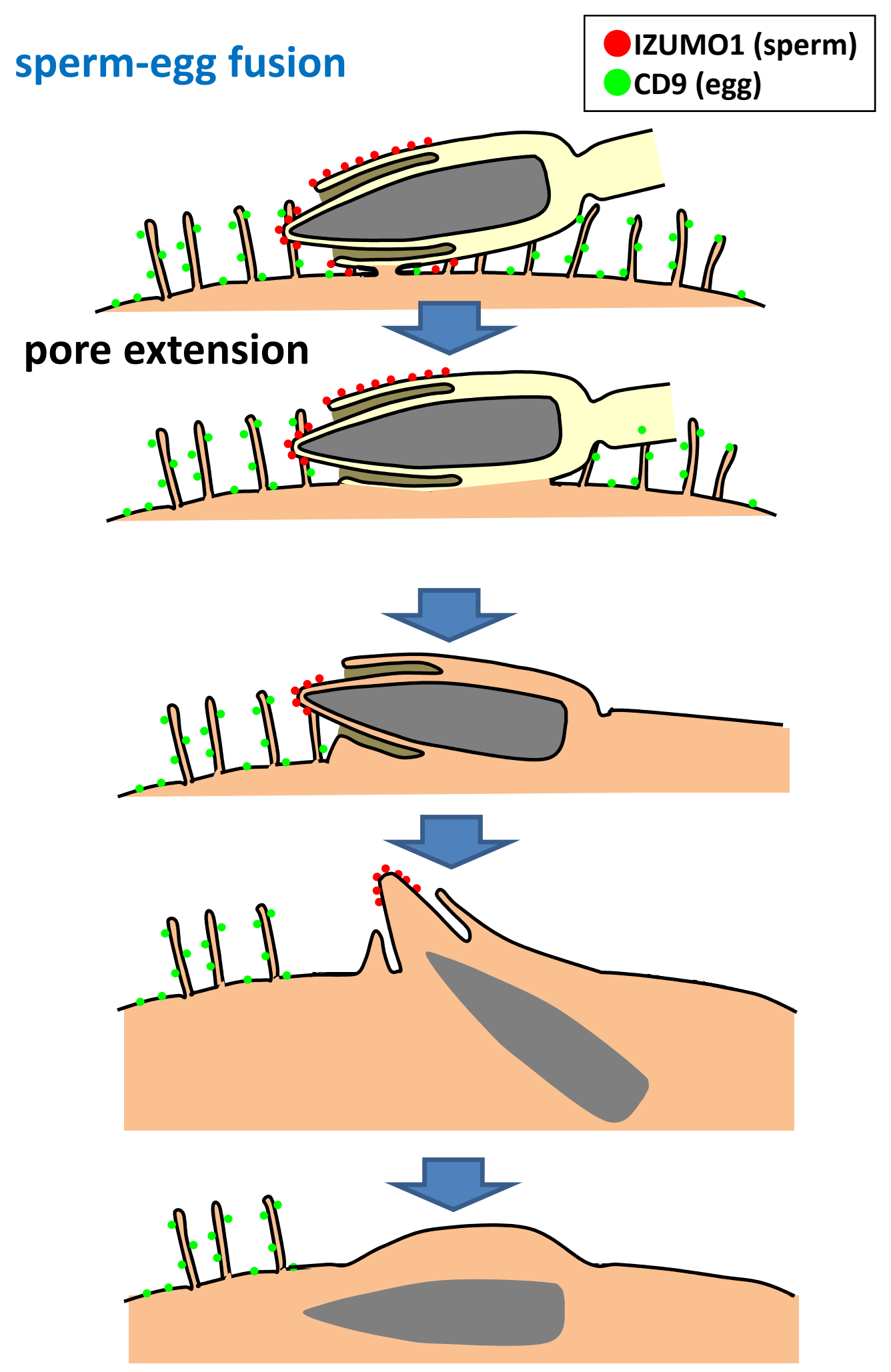

Fig. S2. An estimated outcome of fertilization by a single cell-cell fusion.

The entire sperm and egg membrane mingle together and no internalized vesicle such as shown in figure $4 \mathrm{C}$ would appear. 
A

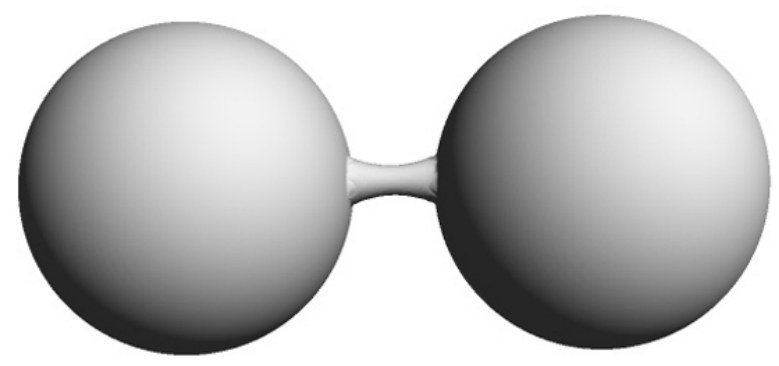

B

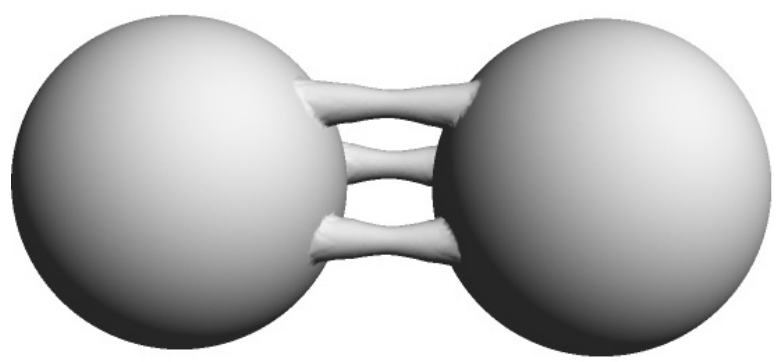

C

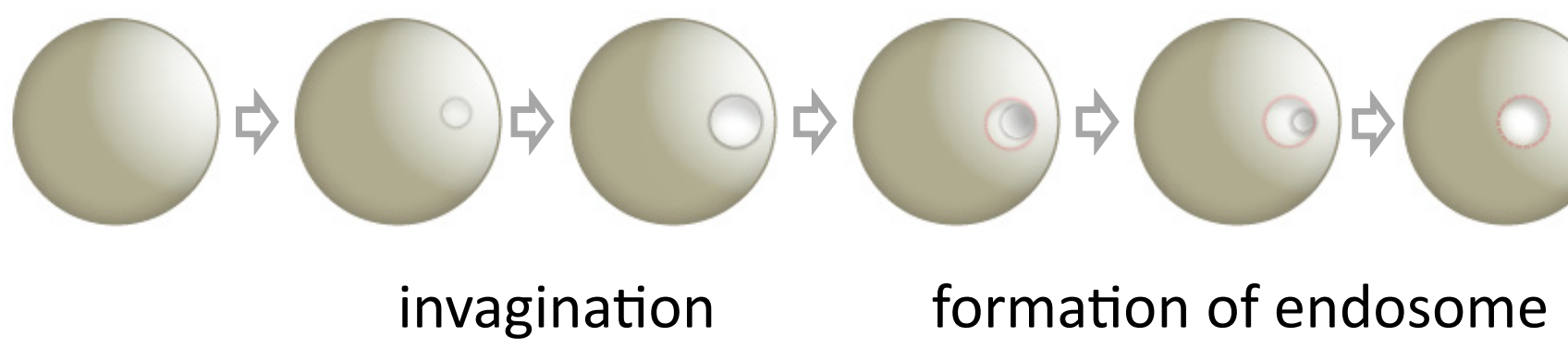

Fig. S3.

(A) A cell-cell fusion joins two membranes into one. (B) The additional fusion at multiple points does not change the number of membrane planes. (C) To form an internalized vesicle, the membrane must fuse in a topographically different manner, with a single membrane divided into two separate parts. 
Table S1. The disappearance of $\mathrm{AC}_{d i m}$ pattern in conditions where fusion was impaired using $C d 9^{-/}$eggs

\begin{tabular}{cccccc}
\hline & \multicolumn{2}{c}{ percentile of distribution pattern of Red-Iz } & & $\begin{array}{c}\text { No. of spermatozoa } \\
\text { egg }\end{array}$ \\
\cline { 2 - 5 } & $\mathrm{AI}$ & $\mathrm{H}$ & $\mathrm{EQ}$ & $\mathrm{AC}_{\text {dim }}$ & examined \\
\hline wild-type & 57.9 & 22.1 & 11.5 & 8.5 & 366 \\
$\mathrm{Cd9}$ & 57.0 & 24.2 & 18.8 & 0.0 & 505 \\
\hline
\end{tabular}

The spermatozoa were mixed with zona-free wild-type and $C d 9^{-/}$eggs. 